\title{
El Diseño Arquitectónico en la Sociedad de la Información y el Conocimiento: Nuevo Paradigma en la Arquitectura
}

\author{
The Architectural Design of the Information Society and Knowledge: New Paradigm in Architecture
}

\author{
Verónica Paola Rossado Espinoza \\ Universidad Ricardo Palma \\ paola.rossado@gmail.com
}

\begin{abstract}
The evolution of technology and the access to information began with the new millennium. Applying the techniques of information and communications in education allows improve the teaching and learning methodology. Influenced by the information society and knowledge, architecture students might be acquiring new architectural design ability. Research on the area of architectural design education, shows that students have an advantage when they use communication and technology. Architectural design is the result of investigation and exchange of ideas, that type of design process could be called a new paradigm in architecture.
\end{abstract}

Keywords: Architectural design; Architecture education; Digital architecture; Architecture paradigm; Information society.

\section{Introducción}

Estamos viviendo en un entorno donde la tecnología está presente en la mayoría de los elementos de nuestro quehacer diario. Los progresos tecnológicos y la información son factores determinantes tanto en la educación, como en el desempeño profesional.

La evolución de las comunicaciones y el intercambio de información comenzó con el nuevo milenio, tal como sucedió con muchas áreas, las TIC incursionaron en la educación, logrando mejorar los procesos de enseñanza - aprendizaje. Bajo la influencia de la llamada sociedad de la información y el conocimiento, surge una nueva metodología educativa a nivel universitario.

Las diversas áreas del conocimiento se han visto favorecidas por el creciente avance tecnológico. El rápido acceso a la información y el desarrollo de las comunicaciones, contribuyen al constante progreso científico. Este desarrollo se ve evidenciado en las investigaciones y proyectos, dando como resultado nuevos descubrimientos. Específicamente en el campo del diseño arquitectónico, la tecnología aporta beneficios en la creación de nuevas soluciones, estructuras innovadoras que contemplan aspectos ambientales, constructivos y una arquitectura sustentable que nuestra sociedad demanda. Además de la modificación de los hábitos de trabajo, mejoras en la representación o el dibujo de planos, la sociedad de la información incorpora variables importantes al diseño arquitectónico. Las TIC, aplicadas a los procesos de enseñanza - aprendizaje en la Arquitectura, han permitido a los alumnos y profesores obtener mejores soluciones, innovadoras y creativas, como respuesta a la demanda de la llamada Sociedad de la Información.

\section{Un Entorno Digital para la Enseñanza.}

El empleo de las herramientas digitales, asociado a los beneficios de la información, ha dado lugar a una nueva forma de producción de conocimientos. Este proceso implica un cambio de metodología en la educación, que involucra tanto a profesores como estudiantes, quienes se han visto obligados a transformar los métodos tradicionales de trabajo para incorporar elementos digitales. Con el objetivo contribuir al desarrollo de una nueva estructura pedagógica, los docentes e investigadores están realizando modificaciones en los planes de clases, obteniendo resultados satisfactorios. El nuevo modelo de aprendizaje, construido en este ambiente virtual, nace en un espacio de cooperación e intercambio de ideas, que combina investigación y producción colectiva, permitiendo la adquisición de nuevos conocimientos. Este espacio virtual de aprendizaje resulta muy atractivo para los jóvenes estudiantes, los motiva a trabajar en forma colaborativa, promueve la investigación y posibilita la creación de nuevas alternativas de solución.

\section{El libre acceso a la información}

Todo sistema educativo debe garantizar que las personas tengan acceso a educación, a los medios y a las comunicaciones; creando condiciones para el progreso y bienestar de la sociedad, haciendo frente a la desigualdad tecnológica. Todos los alumnos tienen derecho a ser parte de estos adelantos y contribuir en la construcción de un mundo sostenible dentro de una sociedad armoniosa.

Esta nueva estructura requiere que las personas desarrollen competencias en función a las necesidades actuales, de manera 
que se preserven los recursos naturales, se evite la degradación de los ecosistemas y se garantice el bienestar humano. Una adecuada educación propicia el desarrollo de los países, el uso adecuado de las herramientas y entornos tecnológicos en forma colaborativa, significará que las personas puedan favorecerse de la información, generando nuevos conocimientos. El aprendizaje es construido sobre la base de una experiencia previa, obtenida mediante la transmisión de saberes.

El acceso e intercambio de experiencias, con el respaldo de profesionales nacionales e internacionales, así como el trabajo colaborativo y compartido, está logrando mejorar sustancialmente los procesos enseñanza, haciendo posible la realización de nuevas investigaciones. La educación es parte esencial en el desarrollo de un país; esta es una etapa donde las nuevas generaciones manifiestan cambios importantes en la transmisión y la gestión del conocimiento.

En el ámbito académico de la Arquitectura, durante la etapa de formación, se evidencian cambios en el desarrollo de los trabajos o proyectos, con un mayor interés del alumnado por desarrollar una arquitectura singular. Las soluciones consideran temas ambientales, respeto por el medio ambiente y la búsqueda de nuevas formas, complejas u orgánicas.

Ante este panorama, surgen algunas interrogantes como: ¿La forma de concebir el diseño arquitectónico, se ha visto enriquecido por el uso de un entorno colaborativo? ¿La sociedad de la información y el conocimiento tiene repercusión en la forma de concebir la arquitectura? ¿Será posible hablar de un nuevo paradigma en el diseño arquitectónico, como resultado del uso de las TIC en la enseñanza?

El presente trabajo intenta esclarecer estas preguntas a través de la observación del trabajo académico de alumnos de años intermedios de la carrera de Arquitectura, y aproximar el concepto de paradigma del aprendizaje del diseño arquitectónico, en el marco de la sociedad de la información y el conocimiento.

\section{La Sociedad de la Información y el Conocimiento}

El término está asociado a la información y a la capacidad que tienen las sociedades de compartir el conocimiento y aprovechar dicha información.

Pertenecemos a la sociedad de la información, el conocimiento y el aprendizaje, esenciales para el progreso y el bienestar de las personas. Gracias a las comunicaciones, se transmite información, lo que genera conocimiento, haciendo posible el desarrollo grupal e individual de las personas. Se está produciendo una fusión entre sociedad de la información y sociedad del conocimiento, aunque su proceso y repercusión recién inician. Según criterio de Peres, "El acceso a las TIC es sólo el primer paso para participar en la sociedad de la información y no refleja en qué medida pueden aprovecharse las posibilidades que ofrece y por ende, sus repercusiones" (Peres, 2009, p.59).

"La competencia (gestión de la información y el conocimiento) conlleva dominio de una serie de conocimientos, habilidades y actitudes, que posibilitan la realización de los procesos previos de adquisición del saber para el desempeño de las profesiones, con apertura del que es de tipo técnico al que tiene un carácter práctico, y de ambos de índole teórica." (García, 2012, p.107). Según el documento publicado por la Comisión Económica para América Latina y el Caribe, CEPAL, respecto a la sociedad de la información, se describe: "El concepto de Sociedad de la Información es muy complejo y su nivel de desarrollo es aún incipiente... esta transformación está impulsada principalmente por los nuevos medios disponibles para crear y divulgar información mediante tecnologías digitales." (CEPAL, 2009, p.11).

Pero todas las sociedades no son iguales, tampoco tienen las mismas posibilidades de acceso a las comunicaciones y a la tecnología, lo que origina una brecha de desigualdad. Del mismo modo, todas las personas no tienen la misma habilidad en el manejo de los conocimientos, o capacidad para comprender, valorar y evaluar la información recibida.

Es importante que el docente estructure y organice las formas de transmisión del conocimiento y la enseñanza con apoyo de las TIC. Se debe promover el uso adecuado de medios virtuales, de manera que sea factible un intercambio de conocimientos, se posibilite la cooperación, el descubrimiento y el autoaprendizaje fuera del aula.

Se espera que esta diferencia o brecha de desigualdad disminuya, siempre apoyados por las instituciones y profesores, motivando el intercambio de conocimiento y la cooperación, que producen nuevos conocimientos, donde la creatividad y la innovación se ven fortalecidas. Citando el informe de la UNESCO, se puede decir que estamos presenciando el advenimiento de una sociedad mundial de la información en la que la tecnología ha superado todas las previsiones, sin embargo, "todavía nos queda un largo camino que recorrer para acceder a las auténticas sociedades del conocimiento" (UNESCO, 2011, p.19).

\section{Paradigma sharista}

A raíz de la aparición del internet y las redes sociales, se produce un cambio de mentalidad, generando un movimiento centrado en la colaboración y el intercambio. Isaac Mao, padre del paradigma sharista, propone una reorientación de los valores de la persona, fundamentado en la neurociencia y el estudio del funcionamiento del cerebro humano. Mao afirma que la sociedad será más eficiente si es capaz de abandonar sus prejuicios y optar por la colaboración para obtener mejores resultados. Considera que la sociedad funciona como un cerebro social súper inteligente, que interconecta a las personas mediante las redes. 
El sharismo pretende transformar el mundo en un cerebro emergente, conectando a las personas por medio de la red. Un cerebro funciona a través de neuronas que forman redes interconectadas, que son capaces de procesar información y aprender. Cada neurona funciona básicamente en el principio de conectar y compartir; la red neuronal tiene la finalidad de compartir actividad e información, incentivando los procesos de pensamiento. A partir de esa actividad, es posible la retroalimentación y la obtención de nuevos aprendizajes. "Cuanto más colectiva sea nuestra inteligencia, más sabias serán nuestras acciones"... "Cuanto menos compartes, menos poder tienes". Mao (2008).

Las personas de la sociedad, son como componentes de un solo cerebro, deben beneficiar a todos sus integrantes, compartiendo información, trasmitiendo y obteniendo nuevos conocimientos. A partir de este paradigma, es posible pensar que el aprendizaje es un compartir de conocimientos que pueden llegar a favorecer a toda la sociedad.

\section{La Información y la Construcción del Conocimiento en los Estudiantes de Arquitectura}

En la Arquitectura y la educación, así como en otras áreas profesionales, los alumnos se ven envueltos dentro de una avalancha de información a través de la web, por lo que deben ser capaces de reconocer y seleccionar los datos de importancia, debido a la falta de veracidad de varios sitios.

Resulta fundamental contar con el apoyo de las instituciones educativas para que los estudiantes tengan acceso a una información válida y garantizada. Un asesoramiento eficaz por parte de los profesores y tutores, favorece la obtención de un buen resultado.

"La correcta apropiación e integración de estas tecnologías a la rutina educativa, la exigencia de adaptación a este contexto o la necesidad de hacer un uso conveniente de los recursos disponibles, son sólo algunos de los desafíos a los que se enfrentan docentes, alumnos e instituciones en este entorno educativo, el cual se está cimentando en las sociedades hipercontectadas". (Ospina, 2012, p.6)

Desde la aparición del primer programa informático para Arquitectura, éste fue relacionado con la representación de planos, siendo entonces considerado como una herramienta que facilitaba el dibujo arquitectónico. La evolución de software especializado, con aplicaciones en la Arquitectura e Ingeniería, permitió extender su uso hacia todas las especialidades del proyecto. El empleo de herramientas digitales pasó a ser parte importante en el desarrollo del proyecto arquitectónico.

\section{Desarrollo del proyecto arquitectónico a nivel universitario}

Resulta difícil reconocer los recursos que utilizan los diseñadores para obtener un buen resultado. En el caso del aprendizaje de Arquitectura, los estudiantes deben adquirir cierto conocimiento y habilidades para enfrentar el diseño arquitectónico y representar adecuadamente sus ideas.

Es importante señalar que, gracias a las TIC aplicadas a los cursos en las diversas áreas académicas, los alumnos tienen la posibilidad de aprender de una manera dinámica, acceder a la información y compartir conocimientos de forma distinta que la realizada hace unos años.

Con el fin de reconocer el efecto que producen las comunicaciones y la sociedad de la información a nivel académico, se realizó una observación del trabajo de un grupo de alumnos de la Facultad de Arquitectura y Urbanismo de la Universidad Ricardo Palma (Lima).

La observación fue aplicada a los cursos de Expresión Arquitectónica, correspondiente a los años intermedios de la carrera, durante un semestre académico. Cabe resaltar que en dicho nivel, los alumnos están en proceso de aprendizaje de diseño, por lo que aún no manejan todas las condicionantes arquitectónicas.

Los alumnos de los grupos observados complementan las clases presenciales con ayuda de plataformas virtuales. Tienen a su disposición la plataforma Moodle, donde el docente realiza el ordenamiento de las sesiones de aprendizaje, coloca los temas a tratar, las ayudas de clase, las evaluaciones, etc. Adicionalmente, debido a que gran parte del alumnado forma parte de una misma red social, el docente utiliza este medio para mantener una comunicación fluida entre los alumnos. Emplea una red privada creada especialmente para el curso, lo hace factible el intercambio de ideas entre sus integrantes y los profesores de una manera inmediata. Por este medio se comparte información de clase, se realizan debates, foros y se publican temas de interés académico.

En los cursos digitales de expresión arquitectónica, que corresponden a los últimos niveles de aprendizaje de dibujo arquitectónico del área, los estudiantes son capacitados en el empleo de herramientas digitales de diseño, quienes realizan la representación de proyectos modelo en dos y tres dimensiones. Luego de concluida la etapa de aprendizaje de los programas, tienen la posibilidad de realizar diseños arquitectónicos propios, en base a un planteamiento o problema inicial.

Los alumnos adquieren la habilidad para representar con exactitud un diseño definido por el docente, pero cuando son enfrentados a un problema de diseño individual, se advierte una serie de dificultades. El docente entonces, proporciona al grupo la información y base teórica necesaria para llevar a cabo el trabajo. 
Los alumnos adicionalmente, consultan sitios web, buscan ideas y ejemplos que puedan proporcionar un aporte teórico o visual al tema.

Se puede apreciar que toda la información adquirida, tanto en el aspecto formal, técnico, ecológico y ambiental, son datos que los alumnos comparten durante todo el desarrollo del diseño. Los gráficos, videos y planos de proyectos similares, son también difundidos en el grupo, esclareciendo dudas e inquietudes mediante la comunicación frecuente, generando un interés en la búsqueda nuevas alternativas.

De esta forma, los alumnos consiguieron la realización de los diseños, aplicando las herramientas digitales y compartiendo la información por medio de las redes. Se logró observar un cambio sustancial en los procesos de creación del diseño, logrando alcanzar un nivel superior de desarrollo y complejidad del proyecto.

\section{Nuevo Paradigma en la Arquitectura}

La experiencia del estudio, hace posible definir cómo la colaboración y el intercambio de información influyeron en el resultado de los procesos de aprendizaje y en los resultados del diseño. Gracias a estos cambios, es posible aproximar un concepto nuevo de diseño arquitectónico a nivel académico, que bien podría llamarse "paradigma en la arquitectura".

Este nuevo concepto puede definirse como: "El proyecto arquitectónico logrado a partir del diseño apoyado por medio de herramientas digitales, creado dentro de un ambiente virtual de colaboración, en respuesta a las necesidades de la sociedad de la información y el conocimiento."

\section{Conclusiones}

Luego de la experiencia del estudio, se pudo observar un cambio sustancial en los procesos de creación del diseño arquitectónico a nivel académico, con la influencia de las comunicaciones. El trabajo colaborativo y el intercambio de información dentro de la realización del proyecto, contribuye a la construcción de este modelo de paradigma o concepto de diseño en la arquitectura.

El nuevo entorno está obligando a los alumnos a investigar y los motiva a trabajar en equipo, incrementando sus posibilidades creativas. La comunicación fluida entre profesor - alumno, permitió el ahorro de tiempo y mejoró la empatía, necesaria para la transmisión adecuada de conocimientos. La dinámica de grupo, la innovación y la creatividad, son factores que se ven fortalecidos ante la aplicación de las TIC en la educación.

\section{Agradecimientos}

Agradezco a mi familia, en especial a mi madre. A mis hijos, Daniel y Gabriel, por su paciencia e interés en todos mis trabajos, ellos son mi mayor fuente de inspiración y esfuerzo. A mis amigos y colegas de trabajo, quienes con su apoyo motivan la realización de nuevas investigaciones y proyectos.

\section{Referencias}

CEPAL (2009). Los Caminos hacia una Sociedad de la Información. Santiago de Chile. Recuperado de: http://www.virtualeduca.org/documentos /2012/cepal_72(2003).pdf

García, L. (2012). Sociedad del Conocimiento y Educación. Librería UNED. Madrid. Recuperado de: http://e-spacio.uned.es/fez/view.php?pid= bibliuned:UNESCO-libros-sociedad_conocimiento

Mao, I. (2008) Sharism: A Mind Revolution. Recuperado de: http://humanismoyconectividad.wordpress.com/2008/12/09/sharis $\mathrm{mo} /$

Ospina, O., Otero, L., Cabrera J. (2012) Educación 2.0: una aproximación a las experiencias educativas mediadas por la tecnología. Universidad del Norte. Barranquilla. Colombia. Recuperado de: http://elaulavirtual.files.wordpress.com/2013/09/educacion_20_una _proximacion_a_las_experiencas_educativas.pdf

Peres, W. Hilbert, M. (2009). Las Sociedad de la Información en América Latina y el Caribe. Desarrollo de las Tecnologías para el desarrollo. Publicación de las Naciones Unidas. Santiago de Chile. Recuperado de: http://books.google.com.pe/books/about/La_sociedad_de_la_ informaci\%C3\%B3n_en_Am\%C3\%A9ri.html?id=pAWDI8x8_EkC\&redir _esc=y

UNESCO (2011) Hacia las Sociedades de Conocimiento. Informe Mundial de la UNESCO. Francia. 